\title{
SCATTERING PROPERTIES OF COMETARY DUST BASED ON POLARIMETRIC DATA
}

\author{
SONOYO MUKAI and TADASHI MUKAI ${ }^{1}$ \\ Kanazawa Institute of Technology \\ Nonoichi, Ishikawa 921, Japan \\ and \\ SEN KIKUCHI \\ National Astronomical Observatory \\ Mitaka 181, Japan
}

\begin{abstract}
Referring to the dust model in Mukai and Mukai(1990), where the scattering by large rough particles and Mie scattering by small particles are taken into account, a phase function of linear polarization of several comets is examined, especially in a region of phase angles $\alpha$ near a maximum polarization. A lower maximum polarization observed in comet Austin(1989c1) than those in comets West(1975n) and P/Halley leads a speculation that a mixing ratio of rough scattering to Mie scattering in comet Austin increases from a sun-comet distance $\mathrm{r}$ of $0.6 \mathrm{AU}$ to $1.2 \mathrm{AU}$. This implies that a shortage of large particles in comet Austin occured in $r<1 \mathrm{AJ}$.
\end{abstract}

\section{INTRODUCTION}

From a study of the previous cometary polarimetry(see e.g. Dollfus et al. 1988), we can draw a simple picture for a phase function of polarization, i.e. "the generalized phase function has a polarization maximum as high as $20-30 \%$ at a phase angle $\alpha=90^{\circ}$, an inversion angle near $20^{\circ}$ and a negative polarization branch at $\alpha<20^{\circ}$ with a polarization minimum several per cent deep"(cited from Dobrovolsky et al. 1986), where a phase angle is defined as a sun-comet-observer angle. Unfortunately, the reliable observations in a wide range of phase angles are very limited, especially in a region of a maximum polarization. Recently, Kikuchi et al.(1990) reported the visible polarization of comet Austin(1989c1) in $12^{\circ}<\alpha<110^{\circ}$. These data initiate us into an analysis of the phase function of polarization near the maximum polarization.

\section{POLARIZATION DATA}

'present address: Dept. of Earth Sciences, Faculty of Science, Kobe University, Nada, Kobe 657, Japan

A.C. Levasseur-Regourd and H. Hasegawa (eds.), Origin and Evolution of Interplanetary Dust, 249-252.

(C) 1991 Kluwer Academic Publishers, Printed in Japan. 
Observed data of linear polarization for 3 comets in a region of maximum polarization are shown in figure 1 . For reference, the data for comet $\mathrm{P} / \mathrm{Halley}$ are also presented. From figure 1, we can summarize the followings: (1) A peak position of a linear polarization is at roughly a phase angle of $90^{\circ}$, although accuracies of the data in $90^{\circ}<\alpha<110^{\circ}$ are quite limited. (2) A degree of polarization in comets Austin and Kohoutek (1973f) is significantly lower in $\alpha>45^{\circ}$ than that in comet West(1975n), even considering a wavelength-dependence and an aperture-dependence of polarization noted in Dobrovolsky et al.(1986) and Michalsky(1981). (3) On the other hand, there are no remarkable differencies among the degrees of polarization for 4 different comets in $\alpha<45^{\circ}$.

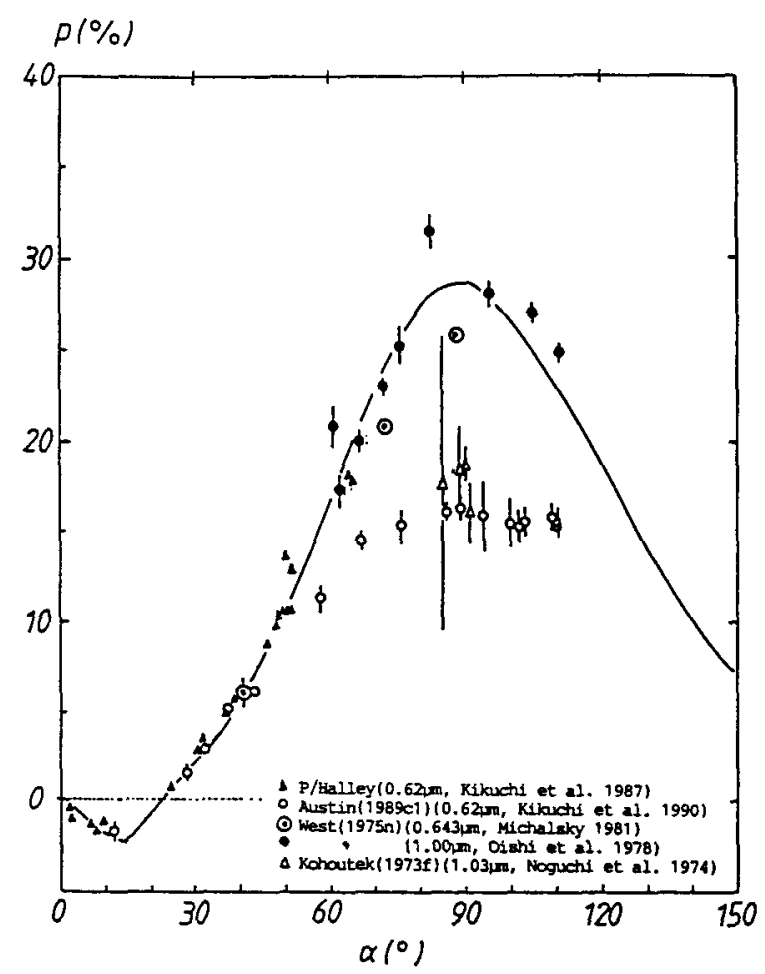

Figure 1. Polarization $\mathrm{p}(\%)$ vs. a phase angle $\alpha$. A number in unit of $\mu \mathrm{m}$ inside round brackets of each observations denotes the effective wavelength. Solid curve is computed based on a mixing model in Mukai and Mukai(1990). The uncertainty in the observed data is shown by error bars.

\section{DUST MODEL}

It has been shown in Mukai and Mukai(1990; to be called MM hereafter) that the scatter- 
ing light by large rough particle reduces the strong negative polarization in the backward direction caused by Mie scattering alone. Simultaneonsly, rough scattering light increases the intensity with decreasing a phase angle(backward enhancement of intensity). These behaviours of rough scattering, accompanying with Mie scattering, lead a favorable tendency to explain the photopolarimetric data observed in the comets in the back scattering region.

The solid curve in figure 1 shows the phase function of polarization computed based on the model having the same parameters as those in MM. It looks that this model can explain the phase function observed in comets P/Halley (Kiknchi et al. 1987) and West(1975n)(Oishi et al. 1978 and Michalsky 1981) not only in the back scattering region, but also in $60^{\circ}<\alpha<110^{\circ}$. However, the maximum polarization reported in comets Kohoutek(Noguchi et al. 1974) and Austin(Kikuchi et al. 1990) shows quite lower value than that expected from the model calculations.

In figure 2, the degrees of polarization computed from Mie theory alone are illustrated for spherical particles with the complex refractive index of $m=1.386-k i$, where $k$ denotes the absorption coefficient of grain material. Here for the size distribution of dust grains the Halley's grain size distribution used in MM was applied. Note that in the mixing model shown as the solid curve in figure 1 , we used the same dust size distribution for Mie particles and $\mathrm{m}=1.385-0.035 \mathrm{i}$.

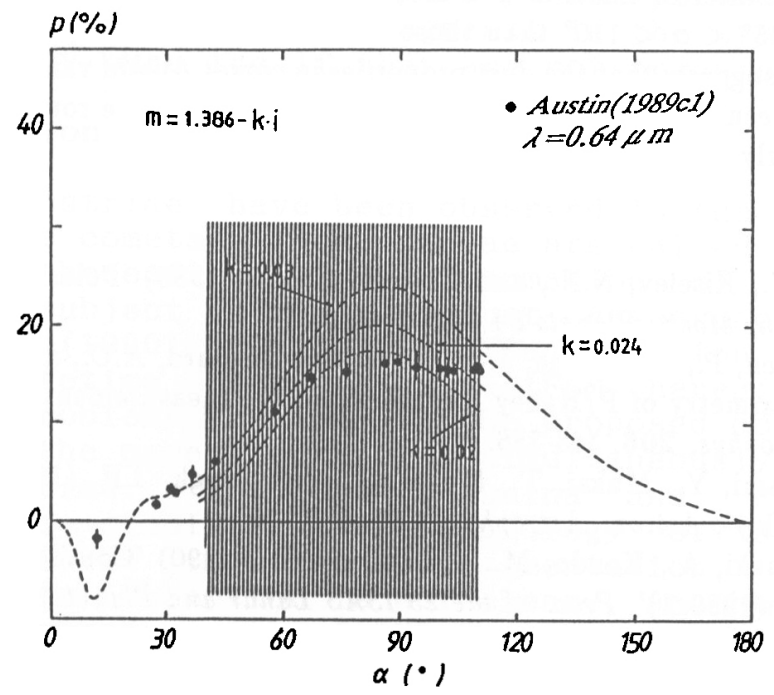

Figure 2. A phase function of polarization. Dashed curves denote the computed results based on Mie scattering alone for the grains with the Halley's dust size distribution and a complex refractive index $\mathrm{m}$. A shaded area presents the region of phase angles where comet Austin(1989 c1) moved inside the Earth's orbit.

It is clear from figure 2 that the phase function computed by Mie theory alone cannot explain the observed results in the back scattering region. The maximum polarization observed near $\alpha=90^{\circ}$ in figure 2, however, gives the hint that the small particles play an important role in this region of phase angles because the light coming from rough scattering 
by large particle moves the peak position of polarization toward the forward direction as reported in the scattering experiments (Killinger 1987). Consequently, we assume that if a mixing ratio $f_{R}$ of rough scattering to total scattering decreases as the phase angle increases, the polarimetric data observed in comet Austin would be explained. Comet Austin moved inside the Earth's orbit when it took the phase angles of $60^{\circ}-110^{\circ}$. Therefore we get a speculation that a relative abundance of large particles in the cometary coma of comet Austin was poor when this comet moved in a sun-comet distance less than $1 \mathrm{AU}$.

\section{CONCLUSIONS}

(1) A phase function of polarization recently observed in comet Austin(1989c1) looks very similar to that detected in comet Kohoutek(1973f), but it is significantly different from those reported in comets West(1975n) and $\mathrm{P} / \mathrm{Halley}$, even taking into account a wavelengthdepencence of polarization and its aperture-dependence. (2) The phase function observed in comets West and $\mathrm{P} / \mathrm{Halley}$ can be well explained by the mixing model of rough large particles and Mie scattering particles with the Halley's dust size distribution and optical constants of $m=1.385-0.035 i$. (3) Although a quite well agreement of polarization in four different comets of interest exists in $\alpha<45^{\circ}$, a significantly lower polarization observed in comet Austin in $45^{\circ}<\alpha<110^{\circ}$ than those in comets West and $\mathrm{P} / \mathrm{Halley}$ appears. This evidence strongly suggests that the dust properties in comet Austin varied with a sun-comet distance $r$. We speculate that in comet Austin a shortage of large rough particles occured in $r$ less than nearly $1 \mathrm{AU}$.

\section{References}

Dobrovolsky, O.V., Kiselev, N.N., and Chernova, G.P. (1986) 'Polarimetry of comets: a review', Earth, Moon, Planets 34, 189-200.

Dollfus, A., Bastien, P., Le Borgne, J.-F., Levasseur-Regourd, A.C., and Mukai, T. (1988) 'Optical polarimetry of $\mathrm{P} / \mathrm{H}$ alley : synthesis of the measurements in the continuum', Astron. Astrophys. 206, 348-356.

Kikuchi, S., Mikami, Y., Mukai, T., Mukai, S., and Hough, J.H. (1987) 'Polarimetry of comet P/Halley', Astron. Astrophys. 187, 689-692.

Kikuchi, S., Okazaki, A., Kondo, M., and Hirata, R. (1990) 'Continuum polarization in comet Austin(1989c1)', Proc. of the 23 ISAS Lunar and Planetary Symp., 39-42.

Killinger, R.T.(1987) 'Photopolarimetrische lasermessungen zur wellenlängen abhängingkeit des lightsterewerhaltens von staubpartikeln aus irdischen und kosmischen materialien' Dissertation(der Ruhr Univ. Bochum)

Michalsky, J.J.(1981) 'Optical polarimetry of comet West 1976VI', Icarus 47, 388-396.

Mukai, S. and Mukai, T.(1990) 'Analysis of photopolarimetric data of comets at small phase angles by rough surface scattering', Icarus 86, 257-263.

Noguchi, K., Sata, S., Maihara, T., Okuda, H., and Uyama, K.(1974) 'Infrared photometric and polarimetric observations of comet Kohoutek 1973f', Icarus 23, 545-550.

Oishi, M., Kawara, K., Kobayashi, Y., Maihara, T., Noguchi, K., Okuda, H., and Sata, S. (1978) 'Infrared observations of comet West(1975n). I. Observational results', Publ. Astron. Soc. Japan 30, 149-159. 